\title{
DOSSIER
}

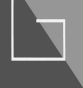

\section{L'affrontement dans la relation de concurrence}

\author{
Le concept d'affrontement \\ concurrentiel s'inscrit \\ dans une approche \\ comportementale de la \\ concurrence, elle-même \\ inscrite dans la métaphore \\ militaire. L'exploration de \\ cette métaphore conduit à \\ définir l'affrontement \\ concurrentiel comme une \\ relation d'opposition \\ médiatisée par les \\ différents marchés sur \\ lesquels les firmes entrent \\ en contact indirect. \\ La nature complexe \\ de l'affrontement \\ concurrentiel amène \\ à proposer de privilégier \\ l'étude de cas approfondie \\ pour mieux comprendre ce \\ phénomène économique. \\ En s'appuyant sur cette \\ méthode, trois voies de \\ recherche sont ouvertes: \\ la première porte sur les \\ déterminants de \\ I'affrontement \\ concurrentiel, la deuxième \\ sur ses modalités \\ et la troisième sur \\ ses implications.
}

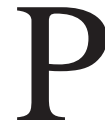
lusieurs auteurs considèrent que les rapports concurrentiels se sont profondément transformés dans les années 1990 (Brahn, 1995; D’Aveni, 1995; Garda et Marn, 1993; Hinthorne, 1996; Young et al., 1996). Aux marchés relativement stables des années de croissance, caractérisés par une concurrence « normale » (Brahn, 1995), se seraient substitués des marchés beaucoup plus disputés, caractérisés par une concurrence « excessive » (Brahn, 1995), « prédatrice » (Hinthorne, 1996) ou « agressive » (D’Aveni, 1995; Young et al., 1996).

Les auteurs s'opposent sur l'attitude à adopter par rapport à cette évolution des marchés. Certains d'entre eux recommandent aux dirigeants de limiter les affrontements, en raison de la dégradation générale des marges qu'ils produisent (Brandemeyer et Schmidt, 1995; Garda et Marn, 1993; Porter, 1982), alors que d'autres considèrent, que, dans un contexte "d'hypercompétition », une entreprise doit être la première à provoquer l'affrontement pour éviter d'être elle-même la cible d'une manœuvre agressive (Craig, 1996; D'Aveni, 1995 ; Young et al., 1996).

Toutefois, si les auteurs divergent sur la nécessité de s'affronter dans la relation de concurrence, ils se rejoignent en ne donnant pas de définition précise de ce concept. Quel sens peut-on accorder à ce concept d'affrontement dans le contexte économique? Quelles 
implications peut-il avoir dans ce contexte? L'objet de cette contribution est de proposer des éléments de réponse à ces questions, en s'interrogeant sur les fondements du concept d'affrontement, sur ses principales modalités et sur ses implications. Cette interrogation permettra de définir clairement les voies de recherche sur ce thème de l'affrontement concurrentiel.

\section{I. - LA RELATION DE CONCURRENCE}

\section{Approche structurelle et approche comportementale de la concurrence}

La concurrence entre les entreprises est un objet d'analyse depuis la naissance et la reconnaissance des sciences économiques comme discipline de recherche. Par suite, il n'est pas envisageable de produire ici une synthèse de l'ensemble des travaux consacrés à cette problématique. En revanche, il est possible de scinder les recherches antérieures en deux grandes approches: l'approche structurelle et l'approche comportementale (Jacquemin, 1994; Scherrer et Ross, 1990).

Dans la première, « les structures productives, les formes actuelles de marché et les modes d'organisation constituent une bonne approximation de ce que devrait être une adaptation efficace à un ordre externe dictés par les caractéristiques de la demande et de la technologie. La compétition est perçue comme un ensemble d'interactions entre les agents qui considèrent comme donnés les comportements d'autrui et les conditions des échanges » (Jacquemin, 1994).
Dans cette approche structurelle, la concurrence est une relation entre des entreprises qui ne se connaissent pas. Il s'agit d'une relation asociale fondée sur la métaphore écologique (Porac et al., 1995). Les entreprises ont pour objectif de créer de la valeur pour les clients avec lesquels elles entretiennent une relation directe. Elles s'influencent indirectement, sans même en avoir conscience, quand elles veulent toucher la même clientèle. C'est cette relation indirecte avec un tiers qu'est le consommateur qui conduit à qualifier leur relation de concurrentielle.

L'intensité de la concurrence est fonction des occasions de recouvrement des offres vis-à-vis d'une même clientèle: plus les entreprises sont, du fait de facteurs structurels, amenées à chercher à satisfaire les même clients de façon exclusive, plus elles sont dans une situation potentielle d'affrontement. Il convient donc, pour déterminer cette intensité de la concurrence, de qualifier précisément les facteurs structurels (Oster, 1990; Porter, 1982). Par exemple, il est possible de déterminer le degré de concentration dans un secteur, en utilisant un indicateur comme l'indice Herfindahl, afin d'en inférer le degré d'intensité concurrentielle (plus cet indicateur est élevé, plus le secteur est concentré et, donc, moins il est concurrentiel). À aucun moment les comportements effectifs des entreprises ne sont pris en compte.

En rupture par rapport à cette approche structurelle, anonyme et mécanique, la concurrence est comprise par de nombreux auteurs comme un problème de comportement des firmes ${ }^{1}$. Cette seconde approche

1. Il est possible de faire remonter ce courant de pensée à Adam Smith (Scherrer, 1980). 
« souligne le rôle actif des agents économiques caractérisés par les asymétries, qu'il s'agisse de l'information ou des dotations de départ. Par leurs actions stratégiques, ces acteurs influencent le choix d'autrui d'une manière favorable à eux-mêmes. Ils se positionnent matériellement et psychologiquement, pour décourager ou pour contraindre les actions et les réactions des rivaux actuels et potentiels; ils modifient de façon crédible les attentes d'autrui grâce à leurs propres engagements, irrévocables ou perçus comme tels » (Jacquemin, 1994).

Après avoir été longuement éclipsées, notamment sous l'influence du premier ouvrage de Porter $(1982)^{2}$, les recherches sur la dynamique comportementale de la concurrence sont actuellement en plein renouveau, notamment dans le domaine du management stratégique (Chen et Miller, 1994 ; Craig, 1996; D’Aveni, 1995; Smith et al., 1992; Young et al., 1996). La rivalité entre les firmes n'est plus considérée comme fonction de facteurs qui s'imposent à elles. Ce sont les dirigeants de ces firmes qui décident, soit de limiter la concurrence, soit d'affronter de façon plus intense leurs concurrents. Loin de s'ignorer, les entre- prises s'identifient comme rivales et leurs comportements sont relatifs à ceux qu'elles identifient comme leurs principaux rivaux. La rivalité concurrentielle peut alors être qualifiée de relation sociale (Porac et al., 1995).

Kuenne (Thomson, 1995) différencie clairement les concepts de concurrence et de rivalité : « la rivalité est distinguée entre les agents par son caractère non anonyme. Dans une interaction de rivalité, chaque agent sait que son action autonome affectera le bien-être de tous ses rivaux sans distinction, et que ces rivaux réagiront pour se protéger [...] Dans un environnement de concurrence, les conséquences externes en termes de bien-être des décisions des acteurs sont si dispersées entre les autres partis intéressés que leurs initiatives ne peuvent pas être identifiées et que des réactions n'ont pas besoin d'être engagées. » Cette conception de la concurrence ne s'inscrit plus dans la métaphore écologique mais dans celle de la guerre militaire: les firmes « manœuvrent », « attaquent » et se « défendent » (Baum et Korn, 1996; Chen et Miller, 1994; D’Aveni, 1995; Le Roy, 1998). Il ne s'agit plus de caractériser les

\begin{tabular}{|l|c|c|c|}
\hline \multicolumn{4}{|c|}{ Tableau 1 } \\
\multicolumn{4}{|c|}{ APPROCHES STRUCTURELLE ET COMPORTEMENTALE } \\
& DE LA CONCURRENCE \\
\hline Niveau de l'analyse & Concepts-clés & Type de relation & Métaphore \\
\hline Structures de secteur & Concurrence & Asociale & Écologique \\
\hline Comportements des firmes & Rivalité & Sociale & Militaire \\
\hline
\end{tabular}

Source: adapté de Le Roy (1998).

2. Pour être tout à fait juste, il faut reconnaître que l'approche comportementale est également présente dans le premier ouvrage de Porter (1982). Ainsi, dans le premier chapitre, Porter considère que « la rivalité existe parce que un ou plusieurs concurrents se sentent poussés à améliorer leur position ou en voient la possibilité. » Toutefois, dans ce premier ouvrage, les développements les plus importants, et ceux qui ont été retenus, s'inscrivent essentiellement dans une approche structurelle de la concurrence. 
déterminants de l'intensité concurrentielle mais de prendre pour objet d'analyse les comportements des firmes, leurs « actions » et « réactions » concurrentielles (Smith $e t$ al., 1992).

\section{Métaphore militaire et relation de concurrence}

L'approche comportementale de la concurrence s'inscrit dans la métaphore militaire (Illinitch et al., 1996; Le Roy, 1998). Or, pour Einhorn et Hogarth (Duhaime et Schwenck, 1985), « les métaphores fournissent des modèles de phénomènes et centrent l'attention directe sur des aspects et des variables spécifiques [...]. Pour être clair, le choix d'une métaphore particulière est crucial, puisqu'elle attire l'attention sur un nombre limité de variables, en excluant les autres ». Comme l'ensemble des métaphores, la métaphore militaire conduit donc à une représentation partielle de l'environnement économique. Cette représentation est fondée sur les modèles militaires qui correspondent aux caractéristiques du contexte militaire.

Or, depuis qu'elle a été « pensée » par Clausewitz, la guerre est définie par les auteurs militaires comme un conflit de volonté qui a pour particularité de se régler par l'emploi de la violence physique. Des actions réciproques sont engagées par les belligérants afin de provoquer des effets destructeurs physiques et/ou psychologiques sur l'adversaire (Le Roy, 1999). Cette relation directe d'échange d'effets de destruction physique est spécifique au contexte militaire. Elle n'a pas d'équivalent dans le contexte économique, sauf à obliger les salariés d'une entreprise à en découdre physiquement avec ceux des sociétés concurrentes pour régler le problème de la répartition des parts de marché. Les relations entre les concurrents ne sont pas directes mais médiatisées par les consommateurs (Le Roy, 1999).

Les comportements des producteurs, comme une innovation ou une baisse de prix, concernent directement les consommateurs. En fonction de ces comportements des firmes, les consommateurs adoptent un comportement d'achat qui affecte les positions des firmes en situation de concurrence. L'interaction concurrentielle est donc une relation indirecte (médiatisée) entre les concurrents: une « attaque » ne peut se comprendre que comme la conséquence indirecte d'une action directe envers les consommateurs. En

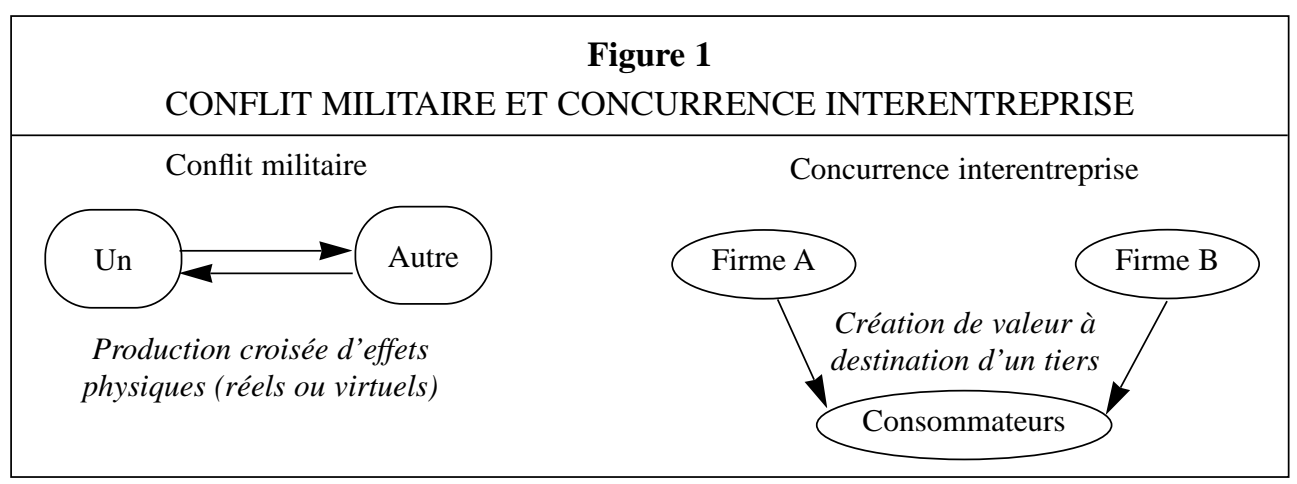

Source: Le Roy (1999). 
d'autres termes, la métaphore militaire conduit à la définition de concepts, comme «l'attaque » ou la « défense », qui sont équivoques dans le contexte économique: ils figurent une relation directe entre les concurrents alors qu'elle est indirecte.

Étant donné cette médiatisation, il n'est pas étonnant que Smith et al. (1992), à la suite de MacMillan et al. (1985), reconnaissent qu'il est difficile d'obtenir une « mesure objective » des manœuvres concurrentielles. Toutefois, ces auteurs ne tirent pas les implications de cette difficulté. Ils évitent le problème en confiant à la presse spécialisée dans un secteur l'identification des interactions concurrentielles. Par exemple, une réaction, ou manœuvre défensive, est considérée comme une donnée à partir du moment où elle est annoncée dans la presse spécialisée de la façon suivante: « en réaction à l'introduction par $\mathrm{X}$ d'un nouveau produit $\mathrm{Y}, \mathrm{Z}$ baisse ses prix de $10 \%$ (Smith et al., 1992). Il semble, au contraire, que, pour comprendre la relation de concurrence, il soit nécessaire de se fonder sur l'ambiguïté que soulève la métaphore militaire, c'est-à-dire approfondir les implications que supposent la médiatisation de la relation concurrentielle. C'est dans cette perspective que nous discuterons dans les paragraphes suivants d'un mode relationnel particulier: l'affrontement.

\section{II. - LA DYNAMIQUE DE L'AFFRONTEMENT CONCURRENTIEL}

\section{Les implications de la nature médiatisée de l'affrontement concurrentiel}

Il existe de multiples définitions en sciences sociales du concept d'affrontement, notam- ment dans des tentatives de distinction entre ce concept et ceux de violence, d'agression ou de conflit. Le plus souvent, l'affrontement est défini comme «le fait d'aller hardiment en face d'un adversaire ou d'un danger » (Timsit-Berthier, 2000), et est associé aux concepts d'opposition et de combat. L'affrontement se produit quand deux individus ou deux groupes sociaux constitués s'opposent ou se combattent pour améliorer leurs positions ou leur bien-être.

L'affrontement concurrentiel peut donc se définir comme une relation indirecte, médiatisée par le consommateur, dans laquelle les firmes rivales s'opposent les unes aux autres pour améliorer leurs positions et leurs performances.

Le caractère spécifique de l'affrontement dans la relation de concurrence, soit sa médiatisation, a des implications fortes pour les acteurs économiques. En effet, cette médiatisation ne permet pas aux managers de se prononcer directement sur les actions de leurs concurrents vis-à-vis des consommateurs. Ces managers sont obligés de produire un jugement sur ces actions, de leur donner un sens, d'inférer de ces actions une intention de la part de leurs concurrents (Heil et Langvardt, 1994). En fonction du sens qu'ils donnent à ces actions, ils vont prendre à leur tour des décisions qui vont avoir des effets indirects sur leurs concurrents.

Une firme peut alors voir sa position indirectement affectée par les comportements d'un concurrent envers les consommateurs sans que ce concurrent n'ait l'intention de lui nuire. L'entreprise qui se sent agressée peut être conduite, en retour, à adopter des comportements avec pour objectif d'affecter la position du rival considéré comme agressif. Cette dynamique conflictuelle 
apparaît clairement dans l'analyse que font Garda et Marn (1993) de l'apparition et de la diffusion de « guerres de prix ». Pour ces auteurs, les guerres de prix sont déclenchées quand une entreprise fait une mauvaise interprétation des actions de ses concurrents.

« Les dirigeants entendent parler des prix de leurs concurrents lorsqu'une personne sur le terrain les alerte: l'entreprise $\mathrm{x}$ est en train de vendre au prix y. Mais il arrive souvent que d'autres informations importantes (par exemple, le fait que le prix n'y soit en vigueur que pendant deux jours et accordé exclusivement à des distributeurs qualifiés, seulement sur des livraisons par camions entiers) n'arrivent jamais à leurs oreilles. Il en résulte que la première entreprise suit le prix y sur toute la ligne. L'entreprise $\mathrm{x}$ voit que son rival propose le prix y à un large marché, ce qui la force, en retour, à proposer ce prix à un plus grand nombre de clients et pour une période plus longue que prévu. Ceci constitue l'un des moyens les plus courants de déclencher et d'amplifier une guerre des prix. L'entreprise qui a mal interprété les données jurera que c'est son concurrent qui a commencé la guerre, tandis que ce dernier verra les choses de manière diamétralement opposée » (Garda et Marn, 1993).

Inversement, une entreprise peut tenter délibérément de déstabiliser un concurrent sans que ce concurrent ne se rende compte de l'intention de nuire de cette entreprise. Baumard et Ibert (1998) montrent ainsi comment un discours rassurant, prononcé notamment dans les assemblées de syndicats professionnels, permet de masquer une politique de déstabilisation des leaders de marchés, menée en l'occurrence par des accroissements de capacités, pendant le temps nécessaire à la mise en place matérielle des investissements.

La nature médiatisée de l'affrontement concurrentiel conduit donc les dirigeants à un processus continu d'observation des actions des firmes rivales à destination des consommateurs, d'interprétation de l'aspect menaçant de ces actions pour les positions et performances de leurs firmes, et d'actions pour s'opposer aux manœuvres qui sont considérées comme susceptibles de remettre en cause ces positions. Ces actions constituent autant de matière à observations, interprétations et décisions de la part de leurs concurrents. L'affrontement concurrentiel ne peut donc être compris que comme un phénomène par nature dynamique et complexe. Ce qui conduit à s'interroger sur la façon dont il est possible de l'étudier.

\section{L'identification des comportements d'affrontement concurrentiel}

La nature médiatisée de la relation d'affrontement concurrentiel ne permet pas, dans une perspective de recherche, de traiter rapidement des questions des déterminants, des modalités et des implications de ce mode relationnel. Il faut nécessairement prendre le temps de mettre en évidence les relations causales entre les comportements d'une firme, leurs effets directs sur les comportements des consommateurs et leurs effets indirects sur les positions des autres firmes. Il s'agit d'identifier les principaux intervenants, de mettre en évidence leurs comportements et les conséquences reliées de ces comportements. La seule méthode de recherche envisageable est alors l'étude de cas approfondie (Baumard, 1996; Le Roy, 1998).

Nous présenterons, pour illustration, une étude de cas portant sur la cession, par la 
Compagnie Saupiquet, de ses activités de conserves de légumes (marque Cassegrain) au Groupe Bonduelle en 1989 (Le Roy, 1998, 2002). Les limites d'une utilisation directe des données de la presse économique apparaissent clairement lors de cette opération. Ainsi, la cession est précédée d'une baisse des prix commentée de façon fort différente. Dans l'Usine Nouvelle du 26 mai 1989, la baisse des prix est qualifiée « d'offensive commerciale du groupe Bonduelle sur les conserves de légumes ». Pour Le Monde du 6 décembre 1989, « la guerre des prix [...], engagée cette année entre distributeurs et fabricants, a durement touché ces derniers ». Dans AGRA Alimentation du 7 décembre, Cassegrain est « victime de la guerre des prix à laquelle elle se livre avec Bonduelle et D'Aucy depuis le début de l'année ».

Une étude de cas approfondie montre que la cession par la compagnie Saupiquet de sa marque Cassegrain au Groupe Bonduelle s'explique par les nombreux investissements dans la création de capacités de production effectués par ce groupe (Le Roy, 1998). En effet, ces investissements ont provoqué un excès d'offre dans le secteur et, par suite, une baisse des prix qui a eu pour conséquence la dégradation du résultat d'exploitation de la Compagnie Saupiquet. Précisément, les évolutions dans le secteur de la conserve de légumes, entre 1986 et 1990, peuvent être résumées de la façon suivante (Le Roy, 1998, 2002):

1. L'objectif de Bonduelle était d'obtenir le leadership sur le marché français des conserves de légumes. Pour atteindre cet objectif, Bonduelle s'est lancé dans un programme d'investissements corporels excédant la croissance de la demande.
2. Ces investissements se sont traduits par un accroissement très important de la production dans le secteur. Cet excès d'offre s'est traduit à son tour par une dégradation des prix jusqu'au troisième trimestre 1989.

3. Ces évolutions sectorielles ont provoqué la décision de cession d'activité. En effet, le positionnement haut de gamme de la marque Cassegrain a conduit les dirigeants de Saupiquet à ne pas baisser leurs prix de vente. Cette décision a entraîné une perte de clientèle si importante que, dès le premier semestre 1989, l'entreprise doit faire face à des déficits d'exploitation intenables dans sa branche conserves de légumes.

Cette dynamique permet de repenser les stratégies de création de capacités de production excessives. En effet, ces stratégies sont communément présentées comme un moyen de dissuader des entrants potentiels. Précisément, en créant des capacités de production excessives, une entreprise est censée signaler à ses concurrents son engagement dans le secteur et sa détermination à s'opposer de façon agressive à une éventuelle entrée (Le Roy, 2002).

L'étude de cas approfondie de la cession de Cassegrain conduit à une autre compréhension de cette stratégie. Dans les situation d'affrontement entre firmes en place dans un secteur, l'investissement permet non pas de menacer d'augmenter, en cas d'entrée d'un nouveau concurrent, la production, mais permet d'augmenter effectivement cette production, afin de provoquer la baisse des prix: l'épreuve de force sur les prix est provoquée par une politique d'investissement dans des capacités de production excessives (voir figure 2). 


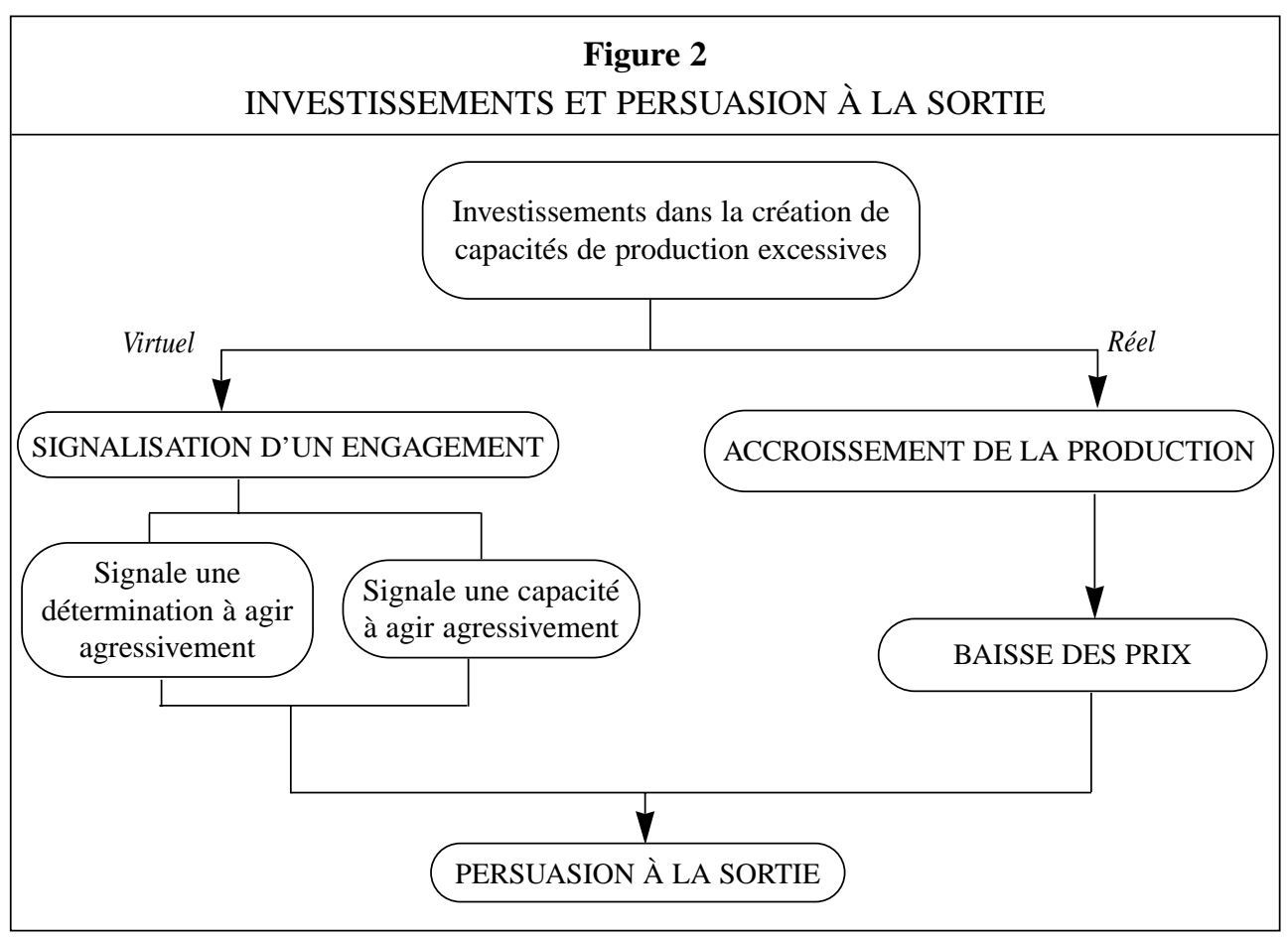

Source: Le Roy (2002).

En même temps que l'augmentation des niveaux de production provoque l'épreuve de forces, la stratégie d'investissement dans des capacités de production excessives permet de convaincre la firme agressée que la baisse des prix correspond à des capacités de production et donc que les prix vont durablement rester faible. Cette anticipation d'une faible profitabilité à moyen terme peut alors conduire la firme agressée à sortir du marché.

Cette illustration conduisant à l'énoncé d'un nouveau cadre de réflexion montre tout l'intérêt de prendre le temps de l'analyse pour comprendre la dynamique des comportements d'affrontement concurrentiel. Cette nécessité est renforcée quand l'affrontement est élargi à l'ensemble des marchés sur lesquels les entreprises sont en relation indirecte.

\section{III. - VERS UNE CONCEPTION ÉLARGIE DU CONCEPT D'AFFRONTEMENT CONCURRENTIEL : LES VOIES DE RECHERCHE}

\section{Une conception élargie de l'affrontement concurrentiel}

Dans les paragraphes précédents, la seule relation indirecte envisagée est celle qui se produit sur les marchés aval. Or les entreprises sont en relation indirecte sur de nombreux marchés. Précisément, une firme qui s'engage dans une stratégie d'affrontement peut agir sur au moins quatre marchés: le 
marché des capitaux, le marché du travail, le marché des approvisionnement et le marché des clients (voir figure 3).

Une première manœuvre indirecte porte sur les détenteurs des droits de propriétés de la firme rivale, sous forme d'OPA inamicale ou de démarchage des actionnaires individuels.

Un deuxième type de manœuvre consiste à agir sur le marché du travail, soit à entrer en contact avec les salariés de l'entreprise rivale. Ces manœuvres consistent essentiellement à proposer des salaires plus attractifs aux cadres ou aux techniciens qui détiennent des compétences-clés. En embauchant ces salariés, l'entreprise prive son rival de compétences essentielles tout en augmentant son propre stock de connaissances (Gardner, 2002).

Une troisième action indirecte revient à agir sur le marché des approvisionnements (Guillotreau et Le Roy, 2000). Une firme peut ainsi chercher à élever les coûts et donc les prix de ses concurrents en établissant des contrats exclusifs avec les sociétés situées en amont de l'industrie. Ces contrats peuvent être de différentes natures, allant du sur-achat d'inputs à l'engagement pur et simple du fournisseur à ne pas vendre aux concurrents, voire à l'intégration du fournisseur.

Un exemple de manœuvre complexe intégrant les fournisseurs est mis en évidence par Le Roy et Guillotreau (2002). Dans l'industrie thonière française (voir figure 4), deux entreprises, Starkist (groupe Heinz) et l'Ona (groupe marocain), entrent dans le secteur en prenant le contrôle de conserveurs français (respectivement Paul Paulet et Pêche et Froid) et des principaux fournisseurs de thon congelé (Cobrecaf et Sovetco). Les capacités d'approvisionnement de ces fournisseurs sont utilisées pour développer une stratégie d'accroissement

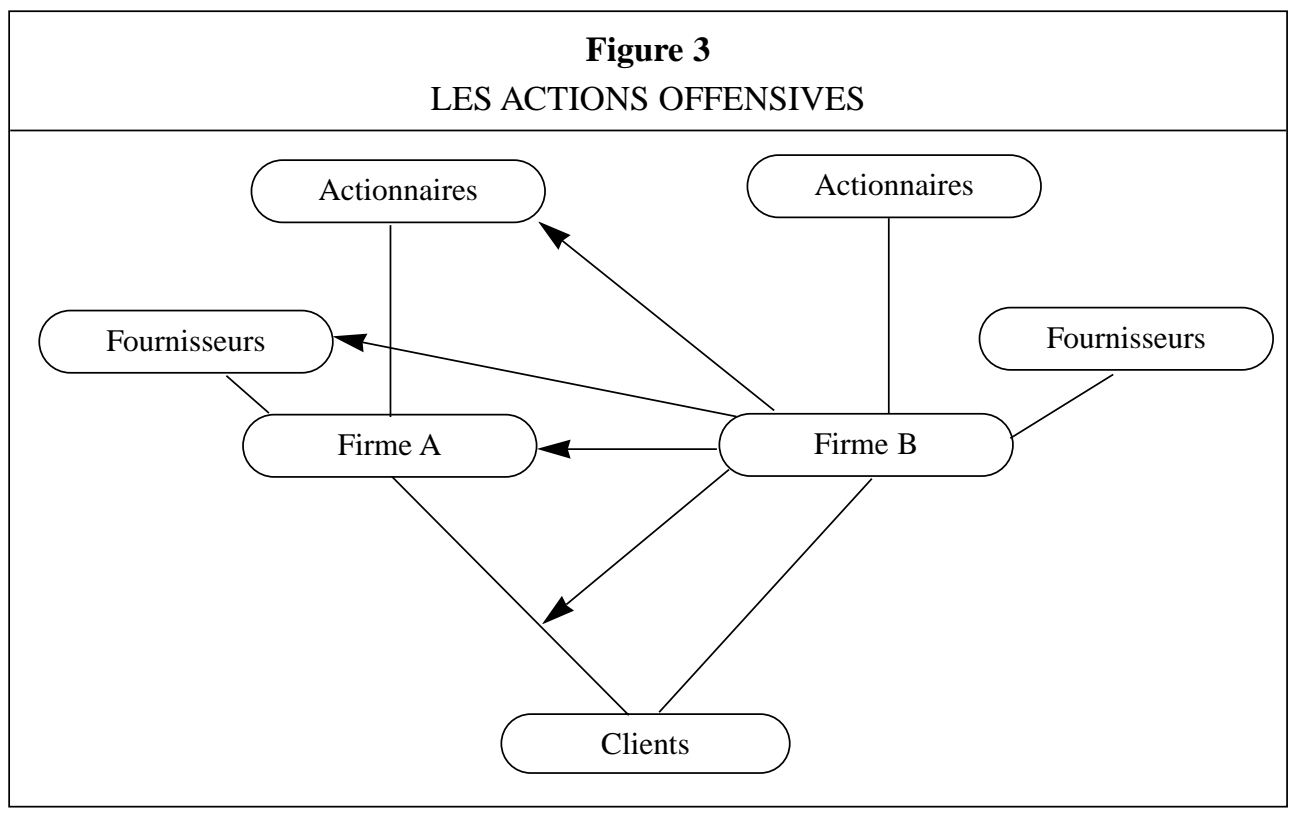

Source: adapté de Crouch (1998). 


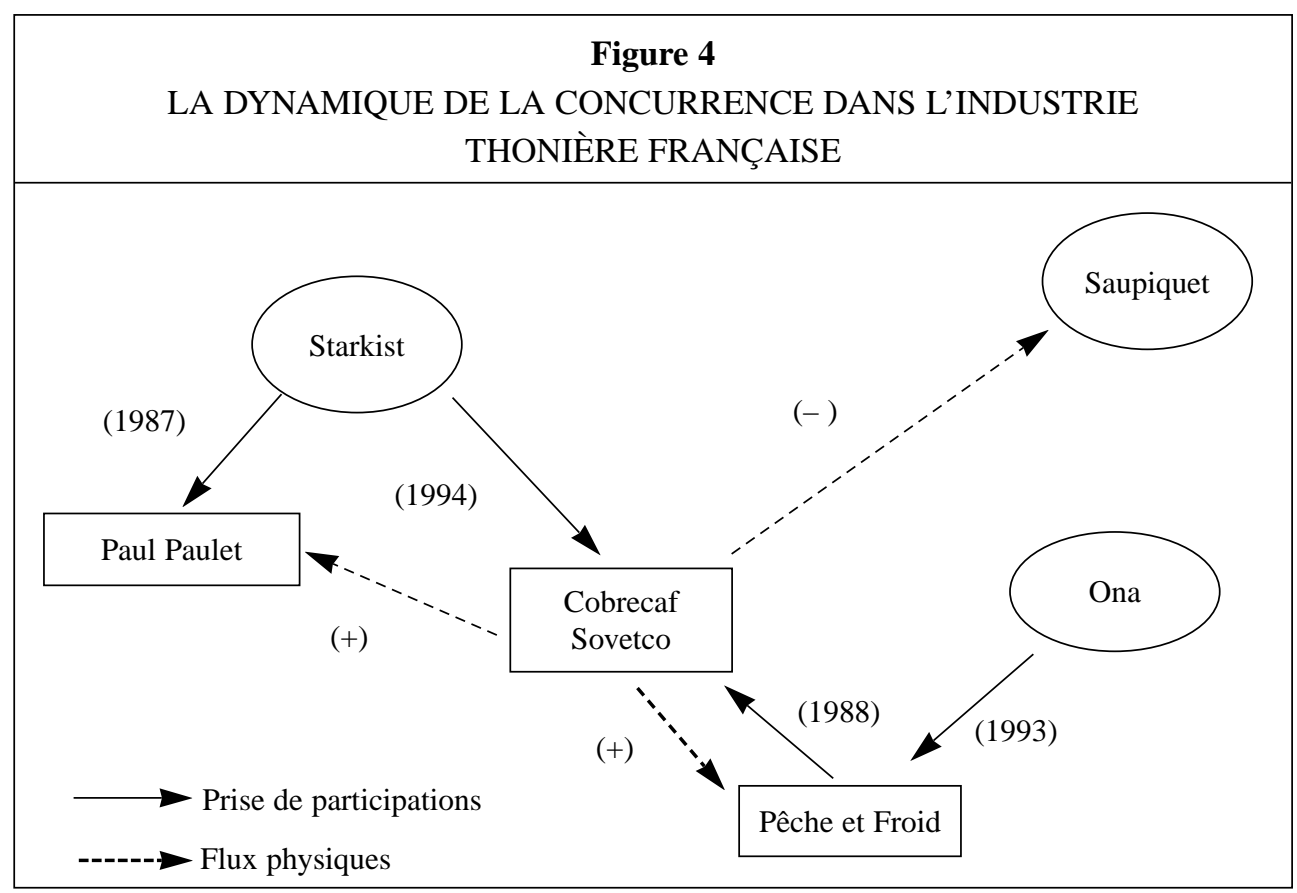

Source: Le Roy et Guillotreau (2002).

des capacités de production des usines de Starkist et de l'Ona. L'ancien leader, Saupiquet, se trouve alors contraint dans ses approvisionnements et, donc, dans sa capacité à développer sa propre production et ses ventes aux même coûts que celles d'Ona et surtout de Starkist.

De façon générale, toutes les relations directes qu'une entreprise entretient avec les parties prenantes (actionnaires, banquiers, fournisseurs de technologie, clients, etc.) peuvent être l'objet d'une manœuvre indirecte agressive de la part d'un concurrent. Une firme peut donc se trouver dans la situation d'être agressée dans le cadre de ses relations avec ses clients, ses fournisseurs, ses actionnaires, etc. De la même façon, elle peut répliquer dans chacune de ces dimensions (voir figure 5).

Dans cette conception élargie, l'affrontement concurrentiel peut se définir comme une relation indirecte, médiatisée par différents marchés sur lesquels les firmes en concurrence s'opposent les unes aux autres pour améliorer leurs positions et leurs performances.

\section{Les voies de recherche}

Trois voies de recherche peuvent être envisagées pour mieux comprendre les relations d'affrontement. La première porte sur les déterminants de ce type de relation, la deuxième sur ses modalités et la troisième sur ses implications. 


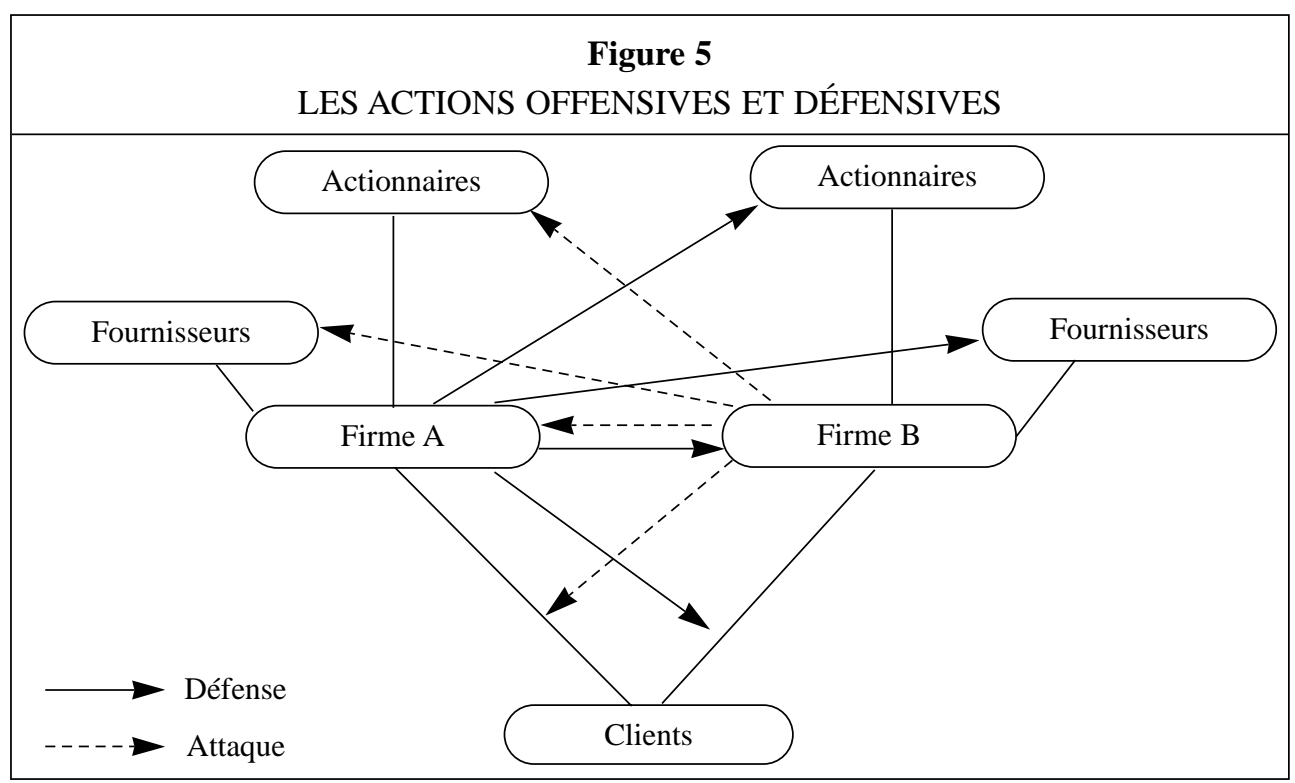

Source: adapté de Crouch (1998).

\section{Les déterminants de l'affrontement concurrentiel}

Il est commun de considérer que l'affrontement est un mode relationnel qui s'instaure dans les contextes structurels de rareté des ressources, et, notamment, dans des contextes de baisse de la demande (Porter, 1982). Or, plusieurs travaux montrent, au contraire, que les contextes de baisse de la demande se traduisent par une plus forte coopération des entreprises alors que les contextes de croissance de la demande se traduisent par une plus forte rivalité (Le Roy, 2002). En effet, les entreprises auraient davantage intérêt à s'entendre et à se concerter quand la demande diminue, notamment en limitant le développement des capacités pour stabiliser les prix. A contrario, une forte croissance de la demande inci- terait les entreprises à des comportements beaucoup plus opportunistes et, donc, à s'affronter pour capter la clientèle supplémentaire.

Il ne semble donc plus possible de réduire les déterminants de l'affrontement à des analyses de nature structurelle. Il convient, au contraire, de s'interroger sur la façon dont un affrontement est le produit d'une interaction concurrentielle. Très précisément, il est nécessaire de travailler de façon approfondie sur les phénomènes de nature psychosociologique qui entraînent le déclenchement d'une relation d'affrontement. Une première recherche dans cette direction montre ainsi l'importance de l'affect, et du sentiment de haine réciproque, dans le déclenchement et l'aggravation d'une situation de guerre des prix (Ibert, 2002). 
Dans cette perspective, la sociologie des conflits est susceptible de fournir des cadres d'analyse jusqu'à présent peu utilisés pour comprendre les interactions concurrentielles (Baumard et Ibert, 1998). Par exemple, l'opposition entre la «théorie des conflits réels », fondée sur les éléments objectifs de la relation de conflit, et la «théorie de la perception de soi », fondée sur les éléments de nature psychosociologique (Hewstone et Grenneland, 2000), pourrait être avantageusement sollicitée pour analyser des relations de forte opposition concurrentielle.

\section{Les modalités de l'affrontement concurrentiel}

La modalité de l'affrontement la plus communément admise et la plus facilement repérable est la guerre des prix (Heil et Helsen, 2001). Une première extension consiste à s'interroger sur le rôle et l'importance d'autres éléments du marketingmix dans la relation d'affrontement, comme le produit ou la publicité (Ailawadi et al., 2001). D'autres extensions, moins répandues, reviennent à introduire dans l'analyse les choix de système technique, et notamment le développement de capacités de production (Ibert, 2002; Le Roy, 2002).

Dans une acception élargie de l'affrontement, il semble nécessaire d'étudier de façon plus approfondie la complexité des relations d'opposition entre les firmes. La diversité des modes d'affrontement mise en évidence dans la figure 5 conduit ainsi à proposer de tenter de repérer et de com- prendre comment les firmes s'affrontent sur les différents marchés où elles sont en contact. Seules de nouvelles recherches dans cette direction permettront de révéler et de mieux comprendre l'intrication des manœuvres et contre-manœuvres complexes d'affrontement ${ }^{3}$.

\section{Les implications de l'affrontement concurrentiel}

Il est commun de considérer que l'affrontement a pour effet de dégrader les marges de l'ensemble des entreprises dans un secteur, que ce soit celles de l'agresseur ou celles de l'agressé (Brandemeyer et Schmidt, 1995; Garda et Marn, 1993; Porter, 1982). Plusieurs recherches récentes amendent sérieusement cette proposition. Par exemple, Le Roy (2003) montre comment deux entreprises leaders de leur secteur ont réussi à éliminer les deux challengers, en déclenchant volontairement un affrontement violent, pour se retrouver en situation de duopole concerté, leur permettant de fixer des prix de vente très avantageux. De la même façon, Baumard et Ibert (1998) montrent comment une entreprise a réussi à renverser à son avantage les positions de marché par un comportement agressif.

Ce type de situation serait si fréquent que D'Aveni (1995) propose d'en faire une nouvelle doctrine stratégique, dans laquelle les firmes qui déstabilisent volontairement leurs concurrents sont celles qui à terme auront les meilleures performances. Entre ce point de vue extrême sur la nécessité d'une stratégie agressive, et le point de vue

\footnotetext{
3. Dans le cas de l'acquisition par Bonduelle de la marque Cassegrain, il est très troublant de constater qu'en même temps que le Groupe Bonduelle se livre à des accroissements de capacités, qui provoquent les pertes opérationnelles de la Compagnie Saupiquet, le principal actionnaire de Bonduelle, le Groupe Paribas, lance une OPA inamicale sur la Compagnie de Navigation Mixte, alors principal actionnaire de Saupiquet et fragilisé par les pertes opérationnelles de sa filiale.
} 
opposé, qui consiste à recommander d'éviter toute forme d'affrontement, il serait nécessaire de mener des recherches sur les conditions de succès et d'échec des stratégies d'affrontement. La double dimension de l'affrontement, à la fois matérielle-économique et psychosociologique, pourrait ainsi être davantage approfondie, notamment en s'interrogeant sur la légitimation d'un comportement agressif, ou, au contraire, sur la perte de légitimité d'une entreprise qui ne respecterait plus les normes de comportement concurrentiel dans un secteur (Baumard et Ibert, 1998; Le Roy et Guillotreau, 2002).

\section{CONCLUSION}

Si de nombreux auteurs reconnaissent la nature de plus en plus conflictuelle des relations de concurrence, peu de travaux de recherche sont centrés spécifiquement sur le concept d'affrontement concurrentiel. $\mathrm{La}$ présente réflexion montre que ce concept s'inscrit dans une approche comportementale de la concurrence, elle-même inscrite dans la métaphore militaire. L'exploration de cette métaphore conduit à définir l'affrontement concurrentiel comme une relation d'opposition médiatisée par les différents marchés sur lesquels les firmes entrent en contact indirect.

La nature complexe de l'affrontement concurrentiel amène à proposer de privilégier l'étude de cas approfondie pour révéler, mettre en évidence et mieux comprendre ce phénomène économique. En s'appuyant sur cette méthode, trois voies de recherche sont ouvertes: la première porte sur les déterminants de l'affrontement concurrentiel, la deuxième sur ses modalités et la troisième sur ses implications. Alors que les modélisations de l'affrontement concurrentiel sont essentiellement développées dans les recherches en sciences économiques, sur la base du calcul micro-économique (Scherrer et Ross, 1990; Tirole, 1988), ces nouvelles recherches devraient permettre aux sciences de gestion de développer des cadres d'analyse qui leur soient propres.

\section{BibLIOGRAPHIE}

Ailawadi K.L., Lehmann D.R. et Neslin S.A., "Market response to a major policy change in the marketing mix: learning from Procter \& Gamble's value pricing strategy", Journal of Marketing, vol. 65, $\mathrm{n}^{\circ} 1,2001$ p. 44-61.

Baum J.A.C. et Korn H.J., "Competitive dynamics of inter-firm rivalry", Academy of Management Journal, vol. 39, $\mathrm{n}^{\circ}$ 2, 1996, p. 255-291.

Baumard P., "Oblique strategist: gaining competitive advantage through conjectural knowledge in unusually aggressive environments", Institut de recherche en gestion, Cahier n 96-05, université de Paris-XII, mai 1996.

Baumard P. et Ibert J., "Knowledge-sharing in Strategically Interdependent Situations: Enacting Collective Assumptions to Escape Retaliation", Academy of Management Meetings, Division Business Policy and Strategy, San Diego,1998. 
Brahn R., "National targeting policies, high-technology industries, and excessive competition”, Strategic Management Journal, vol.16, Special Issue, 1995, p.71-91.

Brandmeyer K. et Schmidt M., «La guerre des prix dans les parfums, de l'autodestruction d'un secteur », Décisions Marketing, n 6, 1995, p. 15-22.

Chen M-J. et Miller D., "Competitive Attack, Retaliation and Performance: an ExpectancyValence Framework", Strategic Management Journal, vol. 15, n 2, 1994, p. 85-102.

Craig T., "The Japanese beer war: initiating and responding to hypercompetition in new product development”, Organization Science, vol. 7, n 3, 1996, p. 302-321.

Crouch A., "Reframing the strategic problem: an accommodation of harmony and belligerence in strategic management", Journal of Business Research, vol. 41, 1998, p. 3-13.

D'Aveni R., Hypercompetition, Paris, Vuibert, 1995.

Duhaime I.M. et Schwenk C.R., "Conjectures on cognitive simplification in acquisition and divestment decision making", Academy of Management Review, vol. 10, n 2, 1985.

Garda R. et Marn M., "Price Wars", The McKinsey Quarterly, n 3, 1993, p. 87-101.

Gardner T.M., "In the trenches at the talent wars: competitive interaction for scarce human resources", Human Resource Management, vol. 4, n 2, 2002, p. 225-237.

Guillotreau P. et Le Roy F., « La guerre du thon ou l'élévation des coûts des concurrents par l'intégration verticale », Annales des Mines, Gérer et Comprendre, n 62, 2000, p. 53-62.

Heil O.P. et Langvardt A.W., The interface between competitive market signaling and antitrust law, Journal of Marketing, vol. 58, n 3, 1994, p. 81-96.

Heil O.P et Helsen K., "Toward an understanding of price wars: their nature and how they erupt", International Journal of Research in Marketing, vol. 18, 2001, p. 83-98.

Hewstone M. et Greenland K., "Intergroup conflict”, International Journal of Psychology, vol. $35, \mathrm{n}^{\circ} 2,2000$, p. 136-144.

Hinthorne T., "Predatory capitalism, pragmatism, and legal positivism in the airlines industry", Strategic Management Journal, vol. 17, n 4, 1996, p. 251-270.

Ibert J., « De la concurrence au conflit irréductible: dynamiques économiques et psychosociologiques», La concurrence : entre affrontement et connivence, Le Roy (ed.), Vuibert, Paris, 2002, p. 27-62.

Illinitch A.Y., D'Aveni R.A. et Lewin A.Y., "New Organizational Forms and Strategies for Managing in Hypercompetitive Environments", Organization Science, vol. 7, n 3, 1996, p. 211-220.

Jacquemin A., «Capitalisme, compétition et coopération», Revue d'économie politique, vol. 104, n 4, 1994, p. 501-515.

Le Roy F., « Dynamique de la concurrence et cession d'activité: le cas de la cession de Cassegrain (Compagnie Saupiquet) au Groupe Bonduelle », Revue Finance-Contrôle-Stratégie, vol. $1, \mathrm{n}^{\circ} 1,1998$, p. 125-143.

Le Roy F., Stratégie militaire et management stratégique des entreprises: une autre approche de la concurrence, Economica, Paris, 1999.

Le Roy F., « Pousser un concurrent à sortir du secteur: la stratégie de création de capacités de production excessives », La concurrence : entre affrontement et connivence, Le Roy (ed.), Vuibert, Paris, 2002, p. 11-26. 
Le Roy F., «Agresser un concurrent pour le sortir du marché: une étude de cas », Revue Finance-Contrôle-Stratégie, à paraître, 2003.

Le Roy F. et Guillotreau P., «Contester la domination des leaders de marché en changeant les règles du jeu: le cas de l'industrie thonière française », Management international, vol. 6, $\mathrm{n}^{\circ} 2,2002$, p. 29-41.

MacMillan I., Mccaffery M.L. et Van Vijk G., “Competitors' Responses to Easily Imitated New Products - Exploring Commercial Banking Product Introductions", Strategic Management Journal, vol. 6, $\mathrm{n}^{\circ}$ 1, 1985, p. 75-86.

Oster S.M., Modern competitive analysis, Oxford University Press, New York, 1990.

Porac J.F., Thomas H., Wilson F., Paton D. et Kanfer A., "Rivalry and the industry models of Scottish knitwear producers", Administrative Science Quarterly, vol. 40, 1995, p. 203-277 Porter M., Choix stratégiques et concurrence, Paris, Economica, 1982.

Scherrer F.M. et Ross D., Industrial market structure and economic performance, Boston: Houghton Miffli, 1990.

Smith K.G., Grimm C.M. et Gannon M.J., Dynamics of competitive strategy, Newbury Park, CA: Sage, 1992.

Thomson W.R., "Principal rivalries", Journal of Conflict Resolution, vol. 39, n 2, 1995, p. $195-223$.

Timsit-Berthier M., «Violence... », Journées de réflexion de l'Afscet, Moulin d'Andé, 18-19 mars 2000.

Tirole J., The theory of Industrial Organization, MIT Press, Cambridge, 1988.

Young G., Smith K.G. et Grimm C.M., “'Austrian' and industrial organization perspectives on firm - level competitive activity and performance", Organization Science, vol. 7, $\mathrm{n}^{\circ} 3$, 1996, p. 243-254. 